\title{
Clearance Failure Rates of Plasmodium falciparum after Treatment among Children (6-11 years) in Kisumu East Sub County
}

\author{
Jomama One Jomama Lual ${ }^{1, *}$, Dan Owino Kaseje ${ }^{2}$, James Aggrey Oloo ${ }^{2}$, Peter Sumba Odada ${ }^{3}$ \\ ${ }^{1}$ Medical Clinic Department, Great Lakes University of Kisumu, Kenya \\ ${ }^{2}$ Tropical Institute of Community Health, Faculty, Great Lakes University of Kisumu, Kenya \\ ${ }^{3}$ Center for Global Health Research, Kenya Medical Research Institute, Kenya
}

Copyright $(C 2015$ by authors, all rights reserved. Authors agree that this article remains permanently open access under the terms of the Creative Commons Attribution License 4.0 International License

\begin{abstract}
Plasmodium falciparum is said to be yet sensitive to artemether-lumefantrine (AL) among all age groups in Kisumu County, despites report of its isolated strain with reduced sensitivity in Kilifi and reported reduced sensitivity to artemisinins in South East Asia. This trial intended to compare Plasmodium falciparum clearance failure rates among children (6-11 years) with uncomplicated falciparum malaria randomly allocated to artesunate-mefloquine or generic artemether-lumefantrine. We conducted a two arms open label randomized controlled trial with a fourteen day follow up and a sample size of 130 children with uncomplicated falciparum malaria in government primary schools of Kisumu East Sub County. This study showed Plasmodium falciparum clearance failure rates significantly higher $(P=0.02)$ with generic artemether-lumefantrine than with artesunate-mefloquine on day one of treatment. However, no significant difference $(P$ $=0.999)$ was observed between the two arms on day seven and day fourteen of treatment. Thus, Plasmodium falciparum was less likely to fail to clear as it responded to artesunate-mefloquine than to generic artemether-lumefantrine on day one of treatment. Nevertheless, it was unlikely to fail to clear as it responded to both artesunate-mefloquine and generic artemether-lumefantrine on day seven and day fourteen of treatment.
\end{abstract}

Keywords Plasmodium falciparum, Artesunate-mefloquine, Clearance Failure Rates, Generic Artemether-lumefantrine

\section{Introduction}

Plasmodium falciparum is said (1) to be yet sensitive to artemether-lumefantrine (AL) among all age groups in Kisumu County, despites report of its isolated strain with reduced sensitivity in Kilifi (2) and reported reduced sensitivity to artemisinins in South East Asia (3). There is incomplete empirical evidence supporting this hypothesis. Firstly, while the sensitivity of Plasmodium falciparum to artemether-lumefantrine has been investigated among under five children and pregnant mothers, it has not been studied among older children (6-11 years) in Kisumu East Sub County. Secondly, although sensitivity of Plasmodium falciparum to artesunate-mefloquine has been established widely in South East Asia (3), it has not been studied among older children in Kisumu County. Lastly, though sensitivity of Plasmodium falciparum to artemether-lumefantrine has been studied (4) in Kisumu, its sensitivity to generic artemether-lumefantrine has not been studied.

Therefore, this trial intended to evaluate the sensitivity of Plasmodium falciparum to artesunate-mefloquine versus generic artemether-lumefantrine among children (6-11 years) in Kisumu East Sub County. It aimed at detecting changes in sensitivity of Plasmodium falciparum. Sensitivity was defined as complete clearance of Plasmodium falciparum from the blood of a child (6-11 years) with uncomplicated falciparum malaria, as it responded to either artesunate-mefloquine or generic artemether-lumefantrine, within seven days of treatment with either artesunate-mefloquine or generic artemether-lumefantrine.

The objective was to compare Plasmodium falciparum clearance rates among children (6-11 years) with uncomplicated falciparum malaria randomly allocated to either artesunate-mefloquine (artequin-300/375 Mepha Switzerland) or generic artemether-lumefantrine (AL by Ipca Ltd (India) in Kisumu East Sub-county.

\section{Materials and Methods}




\subsection{Study Area}

This study was conducted in Kisumu East Sub County, Nyanza province. Malaria transmission is high and perennial in Kisumu, with peak transmission after the rains April June and September- October. $P$. falciparum is the dominant malaria species (5).

\subsection{Study Method Design}

This study was designed as an open label randomized controlled superiority trial (RCT) with a 14 day follow up.

\subsection{Primary Outcome}

The primary treatment outcome was day 14 parasite clearance failure rates. Clearance failure rate was defined as the speed at which peripheral parasite counts are undetectable by microscopy.

\subsection{Secondary Outcomes}

The secondary treatment outcomes were day 1 parasite clearance failure rates and day 7 parasite clearance failure rates.

\subsection{Target Population}

The target population for this study was children (6 - 11 years) in government primary schools in Kisumu East Sub County. Children (6-11 years) in selected government primary school whom no informed parental consent had been provided were excluded from the study. Children (6-11 years) in selected government primary schools who had signs of complicated falciparum malaria, allergic to the drugs, history of mental illness or were severely ill or already taking any medicines for malaria were ineligible for this study.

\subsection{Ethical Consideration}

The study was approved by Great Lakes University of Kisumu and ethical approval certificate was obtained from Ethical Review Committee of great Lakes University of Kisumu. Further study permission was obtained from the Kenya Ministry of Education. The head teachers of the selected government primary schools were also requested for study permission. Informed written parent consent was obtained before Plasmodium falciparum testing was conducted. Before parents decided to consent, the parents were met and discussed with the principal research in their respective schools. In these discussions both benefits and potential side effects of both artesunate-mefloquine and generic artemether-lumefantrine were explained to both teachers and parents. Besides, the potential drugs side effects were included in the consent form. Confidentiality of collected data was ensured by allowing data access only to relevant health institutions and authorities.

\subsection{Inclusion Criteria}

Children in randomly selected government primary school:

- Age 6-11 years old.

- Axillary body temperature less than 38.0 degree Celsius.

- Confirmed mono infection with Plasmodium falciparum.

- Parasitaemia of $40-100,000$ asexual parasites per micro litre.

- Signed parental consent form was eligible for this trial.

\subsection{Sampling Technique}

Using systematic random sampling technique, 4 government primary schools were selected from a list of government primary schools in Kisumu East Sub County. Six to eleven year old children from pre-school, standards one, two, three and four were screened for Plasmodium falciparum malaria. Assuming a malaria prevalence of $20 \%$ for children aged 6-11 years, a total of $(100 / 20 \times 100)=$ 500 pupils were to be screened. But due to low prevalence of parasitaemia among children (6-11 years) in some schools, a total of 1300 children were screened in order to be able to achieve the required study sample size.

\subsection{Sample Size}

A sample size of 112 children as needed to detect a difference of $9 \%$ between artesunate-mefloquine arm and generic artemether-lumefantrine arm: 56 children at each arm of study. Due to expected difficulty with older children's follow up in the community, a large percentage of lost to follow up was necessary; so, 35\% lost to follow up was anticipated. Adding 35\% lost to follow made a total sample size of 152 children: 76 children at artesunate-mefloquine arm and 76 children at generic artemether-lumefantrine arm. However, after randomization and before drugs administration we lost a total number of 22 children: 2 at generic artemether-lumefantrine arm (both due to withdrawal of parental consent) and 20 at artesunate-mefloquine arm (11 due to withdrawal of parental consent, 3 due to late disclosure of history of mental illness, 6 developed signs of complicated falciparum malaria before drug administration). We enrolled and administered study medicines to a total of 130 children: 56 at artesunate-mefloquine arm and 74 at generic artemether-lumefantrine arm. None of the children enrolled was lost to follow up after drug administration.

\subsection{Data Collection Method}

In estimating parasitaemia, both thin blood film and thick blood film were prepared. Parasites' counting was started with thin blood film and switched to thick blood film as thin 
blood film parasite counts declined towards 1 parasite per 1000 red blood cells. In the thick blood film parasites were counted per 200 white blood cells. The study's research work consisted both of field work and laboratory work.

In the field, the clinical research team was composed two (2) Clinical Officers whose job was to take history, examined the child and refer serious cases to the Pediatrician Medical Doctor. Then three (3) qualified and experienced (in clinical research) nurses who were responsible for taking weight, vital signs and drug administration. Four (4) laboratory technicians who were responsible for taking blood samples, preparing thin blood film and thick blood film. There was one (1) logistics officer who was responsible for logistics. There was one (1) driver who was responsible for transporting staff and logistics. There were four (4) community mobilizers who were responsible for mobilizing children, parents and teachers in schools.

In the laboratory, there were five laboratory technicians who were responsible for staining blood slides and blood slides reading: two (2) first readers, two (2) second readers and one (1) tie breaker. A blood slide reader read no more than fifty blood slides per day. Lastly, the principal researcher supervised the clinical team and from time to time consulted with his professors: Kaseje Dan, Oloo James and Odada Sumba.

In the event of any serious illness, the child was to be treated and managed at St. Luke Hospital by a Pediatrician Medical Doctor. An adverse event was defined as any event occurring new or worsening from baseline. However, no adverse event was observed or reported during the study period.

Although the evening dose of generic artemether-lumefantrine was not directly observed because it was not practical to do so in primary day schools, administration of drugs was closely monitored and directly observed by nurses on daily basis in the morning until the complete 3 day course of both artesunate-mefloquine and generic artemether-lumefantrine was taken by the enrolled child. During each scheduled visit the enrolled children were interviewed and examined by a clinician. Findings from the interview and physical examination were recorded on a case report form (CRF). No abnormality was noted.

\subsection{Data Collection Tools and Quality Assurance}

Data were collected and recorded on data collection forms. Data recording forms were pre-tested and dry run (rehearsal) was done with the clinical team one day before field work started. Feedback observations from pre-testing and rehearsal processes were used to improve on the data collection tools and processes.

\subsection{Data Collection Procedure}

Each selected government primary school generously provided a spacious hall for screening the children for falciparum malaria. In each spacious hall, we arranged and divided the hall into four work stations: station one (1) was for registration; station two (2) was for history taking and physical examination; station three was for taking blood sample and blood slides preparation; and station four was for drugs administration. The community mobilizer with the assistance from school teachers brought children to the screening hall first at registration desk where nurses registered took body weight in kilograms and axillary body temperature in degree Celsius. Then nurses forwarded the registered children to station two (2) where clinical officers took history and conducted physical examination. Here at station two (2), children were often dropped if they failed to meet the study inclusion criteria. The children who met inclusion criteria at station two were sent to station three (3) where the laboratory technicians took the peripheral blood sample from the finger first for rapid diagnostic test and later for a blood slide for microscopy if the rapid diagnostic test's result was positive for falciparum malaria. In taking the blood specimen, the child was identified correctly using full names, serial number, class and school. The procedure was introduced to the child by creating good rapport and explanation. Then the puncture site was identified: side puncture at fingertip was the most preferred. The selected puncture side was swabbed with $70 \%$ alcohol and left for few seconds to dry. Finally, a good puncture was made and first drop of blood was wiped using dry cotton wool. Then blood was taken to prepare thin blood film and thick blood film. Each rapid diagnostic test sample and blood slides were clearly labelled with a child's serial number, name of school and date of sample collection. Prepared blood slides were covered and protected from dust and flies. While awaiting the rapid diagnostic test result the tested children were given sweets. But during blood sample for microscopy they were given milk cream biscuits.

In the laboratory, using $10 \%$ Giemsa stain, a number and density of Plasmodium falciparum was estimated by counting first against 1000 red blood cells in the thin blood film and later in the thick blood film against 200 white blood cells. The giemsa stain used was manufactured by Carol Clinical Systems M-46, Phase III B, Vernal Industrial Estate Verna Goa 403722 India. Lot no GMS 1064. ISO 9001 2008; ISO 13485 2003. It was manufactured on January, 2013 and was to be expired on December, 2017. It was giemsa $100 \%$ in $500 \mathrm{ml}$ bottle from which a working solution of $3 \%$ was prepared. Smeared blood slides were left to air dry. Thin blood films were fixed using absolute methanol. Known positive and negative blood slides were stained concurrently for quality control of the stain that was to be used. The first reader read the slide; the second reader confirmed the first reader's reading; and the third reader read the slide to tie break in case of any substantial discordance between the first reader and the second reader. Where a blood slide for microscopy was read only by the first reader and second reader, the second reader's reading was preferred. Where a blood slide was read by the first reader, second reader and third reader, the third reader's reading was believed. Since the technicians in the laboratory did not go to the field, they 
did not know the drugs allocated to the children whose blood slides they were examining for Plasmodium falciparum. Thus, they were blinded.

If the blood slide for microscopy was positive then the positive children were sent to station four (4) for drugs administration after which they were provided with additional milk cream biscuit. Since we could not guarantee the hygienic status of locally available fatty foods, the milk cream biscuit was thought to be a better source of fats especially for children randomly assigned to generic artemether-lumefantrine.

Random sequence of numbers that corresponded to artesunate-mefloquine and artemether-lumefantrine were generated using a computer random number generator. Children were assigned to either artesunate-mefloquine or artemether-lumefantrine using the pre-printed sequence. Enrolled children were followed up for 14 days. Day zero (0) was defined as the first day of testing and taking the drug. Re-assessments and subsequent blood slide sampling were conducted on day one (1), day seven (7) and day fourteen (14) of treatment.

Artesunate-mefloquine was taken in 3 doses. Each dose was two tablets. The first dose of two tablets was taken after initial diagnosis (day 0), the second dose after 24 hours (day 1) and the third dose after 48 hours (day 2). For a child weighted 15-24 $\mathrm{kg}$ the dose of generic artemether-lumefantrine was, on day zero, 2 tablets at 0 hour and 2 tablets after 8 hours, 2 tablets in morning and 2 tablets in evening of day one, 2 tablets in morning and 2 tablets in evening of day two. For $25-34 \mathrm{~kg}$ child the dose was 3 tablets at 0 hour and 3 tablets after 8 hours on day zero; 3 tablets in morning and 3 tablets in evening of day one; 3 tablets in morning and 3 tablets in evening of day two.

\subsection{Data Management}

Immediately after field and laboratory work, data was entered into Statistical Package for Social Sciences (SPSS) version 21.0 on daily basis by the principal researcher. Consistency and completeness of data was ensured and data was cleaned. All the positive malaria slides were well keep. Each positive slide was entered individually: no merging was done during data entry.

\subsection{Data Analysis}

Data was analysed using SPSS version 21.0. Descriptive statistics were used to describe characteristics of research participants. To compare parasite clearance rates between the generic artemether-lumefantrine versus artesunate-mefloquine, chi square test was used with p-value to show significance where cell count values were greater than 5. T test was used where cell count values were less than 5. Binary logistic regression analysis was used to predict clearance and control for confounding factors (such as age). In order to compare probabilities of clearing parasites, the probabilities were derived and plotted in excel. Two samples mean test was used to compare geometric mean density of Plasmodium falciparum; $95 \%$ CI was used to denote significance. To compare parasite clearance failure rates between the generic artemether-lumefantrine versus artesunate-mefloquine, chi square test was used with p-value to show significance where cell count values were greater than 5 . $T$ test was used where cell count values were less than 5. In this case, $95 \% \mathrm{CI}$ was used to show significance.

\subsection{Statistics}

Chi square test was used to compare proportions in categories of sex and area; independent sample test to compare means of age, body weight and body temperature; and Wilcox rank test to compare medians of parasite density.

\section{Results}

\subsection{Study Participants}

This study enrolled children (6-11 years) from four government primary schools in Kisumu East Sub County. 56 of them were males while 74 were females. 7 children were enrolled from Wandiege primary school; 33 from St. Mark, 43 from Buoye and 57 from Migingo.

\subsection{Socio-demographic Characteristic of Study Participants}

In this study, a total of 130 children from age 6 to 11 years, in four primary schools that were randomly selected from government primary schools in Kisumu East Sub County, met the inclusion criteria and were enrolled. Of these, 56 were randomly allocated to artesunate-mefloquine's arm while 74 were randomly assigned to generic artemether-lumefaantrine arm. Socio-economic as well as clinical characteristics of study participants were compared in order to assess the degree to which randomisation was achieved. These characteristics included sex, area of school, age, weight, and temperature and parasite density. Table 1 has details on socio-demographic and clinical characteristics of study participants.

\subsubsection{Sex}

Among the 56 children (6-11 years) randomly assigned to artesunate-mefloquine $43 \%$ were males while $57 \%$ were females. There was a difference of $7 \%$ between the proportions of males and females. Many women compared to men were randomly assigned to artesunate-mefloquine. However, the difference in the proportions was statistically insignificant $(P$-value $=0.554)$ when compared using Chi square test. The Chi square statistical test was used because counts in each cell were greater than 5. The difference between the proportions of females and males in artesunate-mefloquine arm was attributed to chance. So, being female or male did not affect the random assignment to 
artesunate-mefloquine.

Of the 74 children C6-11 years) randomly allocated to generic artemether-lumefantrine $58.6 \%$ was females while $43.2 \%$ was males. A difference of $15.4 \%$ between males and females was seen. Many women compared to men were randomly allocated to generic artemether-lumefantrine. But the difference in the proportions was statistically insignificant $(P$-value $=0.554)$ when compared using Chi square test. The Chi square statistical test was used because counts in each cell were greater than 5 . The difference between the proportions of females and males in generic artemether-lumefantrine arm was attributed to chance. Therefore, being female or male did not influence the random allocation to generic artemether-lumefantrine. Thus, in sex, randomisation was achieved.

\subsubsection{Area of school}

Of the four primary schools, two schools were selected from urban while two were selected from rural areas of Kisumu East Sub County. Out of 56 children randomly allocated to artesunate-mefloquine arm, $32 \%$ was enrolled from urban schools while $68 \%$ was enrolled from rural schools. A difference of $36 \%$ was observed between urban schools and rural schools. Many children, from rural schools compared to urban schools, were allocated to artesunate-mefloquine. The observed difference between rural schools and urban schools was statistically insignificant $(P$-value $=0.06)$ when tested using Chi square test. The Chi square statistical test was used because counts in each cell were greater than 5 . The difference in the proportions of rural children and urban children allocated to artesunate-mefloquine was attributed to chance. Therefore, randomisation was achieved in area as a demographic variable of study participants.

\subsubsection{Age}

The overall mean age of study participants was 9: while it was 8 at the artesunate-mefloquine arm and 10 at generic artemether-lumefantrine arm. The children randomly assigned to artesunate-mefloquine were much younger while the children randomly allocated to artemether-lumefantrine were much older. The mean age was significantly different $(P$-value $<0.001)$ when it was compared using independent sample T-test. The independent sample T-test was used because the age distribution was normal. The difference in mean age was not attributed to chance. So randomisation, in age, was not achieved.

\subsubsection{Weight}

The overall mean weight of study participants was $21 \mathrm{~kg}$. While the mean weight was $18 \mathrm{~kg}$ at artesunate-mefloquine arm, it was $22 \mathrm{~kg}$ at generic artemether-lumefantrine arm. Children assigned to artesunate-mefloquine weighted less compared to those children allocated to generic artemether-lumefantrine. The mean weights were significantly different when compared using independent sample T-test. The independent sample T-test was used because the weight distribution was normal. The difference in mean weight was not attributed to chance. So randomisation, in weight, was not achieved.

\subsubsection{Body temperature}

The overall mean body temperature was 37.4 degrees Celsius. Whereas mean body temperature was 37.352 degrees Celsius at artesunate-mefloquine arm, it was 37.376 degrees Celsius at generic artemether-lumefantrine arm. There was a difference of 0.02 in mean body temperature between those children randomly assigned to artesunate-mefloquine and those children randomly allocated to generic artemether-lumefantrine. The children randomly allocated to generic artemether-lumefantrine had slightly higher body temperature compared to children randomly assigned to artesunate-mefloquine. The different in mean body temperature was statistically insignificant when compared using independent sample mean t-test. The independent sample T-test was used because the body temperature distribution was normal. The difference in mean body temperature was attributed to chance. So randomisation, in body temperature, was achieved.

\subsubsection{Parasite density}

The overall parasite median (per micro-litre) was 160 parasites per micro-litre. While the parasite median was 140 parasites per micro-litre at artesunate-mefloquine arm, it was 160 parasites per micro-litre at generic artemether-lumefantrine arm. A difference of 20 parasites per micro-litre was observed. Children randomly assigned to artesunate-mefloquine arm had less parasites per micro-litre compared to children randomly allocated to generic artemether-lumefantrine. The difference in parasite median was statistically insignificant $(P$-value $=0.410)$ when compared using Wilcoxan rank sum test. The Wilcoxan rank sum test was used because the parasites density distribution was skewed. The difference in parasite median was attributed to chance. So randomisation, in parasites density, was achieved. 
Table 1. Socio-demographic and clinical characteristics of study participants

\begin{tabular}{|c|c|c|c|c|}
\hline & 0verall & $\begin{array}{l}\text { Artesunate-mefloquine } \\
(\mathrm{n}=56)\end{array}$ & $\begin{array}{l}\text { Artemether-lumefantrine } \\
(\mathrm{n}=74)\end{array}$ & P-value \\
\hline Sex* & $\begin{array}{l}56(43.1) \\
74(56.9)\end{array}$ & $\begin{array}{l}24(42.9) \\
32(57.1)\end{array}$ & $\begin{array}{l}32(43.2) \\
42(56.8)\end{array}$ & 0.554 \\
\hline Area* & $\begin{array}{l}31(23.8) \\
99(76.2) \\
\end{array}$ & $\begin{array}{l}18(32.1) \\
38(67.9)\end{array}$ & $\begin{array}{l}13(17.6) \\
61(82.4)\end{array}$ & 0.063 \\
\hline Age (years)* & $\begin{array}{l}8.88 \\
9.00 \\
6,11\end{array}$ & 7.93 & 9.61 & $<0.001$ \\
\hline Weight (kg)* & $\begin{array}{c}20.528 \\
22.00 \\
15.0,26.4 \\
\end{array}$ & 18.213 & 22.281 & $<0.001$ \\
\hline $\begin{array}{c}\text { Temperature } \\
\text { (Degree } \\
\text { Celsius)* }\end{array}$ & $\begin{array}{c}37.365 \\
37.400 \\
37.0-37.9 \\
\end{array}$ & 37.352 & 37.376 & 0.575 \\
\hline $\begin{array}{c}\text { Parasite } \\
\text { density (per } \\
\text { micro-litre)* }\end{array}$ & $\begin{array}{c}160 \\
40,18440\end{array}$ & 140 & 160 & 0.410 \\
\hline
\end{tabular}

* Males n (\%), females n (\%). * Urban n (\%), rural n (\%). *Mean, median, minimum, maximum age. *Mean, median, minimum, maximum body weight. * Mean, median, minimum, maximum body temperature. $*$ Median, minimum, maximum parasite density;

\subsection{Comparison of Rates of Clearance Failure}

The rates of clearance failure were compared between artesunate-mefloquine arm and generic artemether-lumefantrine arm on day 1 , day 7 and day 14 of treatment.

\subsubsection{Day 1 rates of clearance failure}

Of the 56 children randomly assigned to artesunate-mefloquine, 5\% had not cleared Plasmodium falciparum from their blood at day one (after 24 hours) of treatment with artesunate-mefloquine while of the 74 children randomly allocated to generic artemether-lumefantrine, 22\% had not cleared Plasmodium falciparum from their blood at day one (after 24 hours) of treatment with generic artemether-lumefantrine.

A difference of $17 \%$ in rates of parasites clearance failure was observed. Children randomly assigned to artesunate-mefloquine had lower rates of clearance failure at day one (after 24 hours) of treatment with artesunate-mefloquine compared to children randomly allocated to generic artemether-lumefantrine at day one (after 24 hours) of treatment with generic artemether-lumefantrine.

The $17 \%$ difference in rates of parasites clearance was statistically significant when compared using Fisher's exact test. The Fisher's exact statistical test was used because counts in some cells were less than 5. Therefore, children randomly assigned to artesunate-mefloquine were less likely to fail to clear Plasmodium falciparum from their blood at day one (after 24 hours) of treatment with artesunate-mefloquine than children randomly allocated to generic artemether-lumefantrine at day one (after 24 hours) of treatment with generic artemether-lumefantrine.

\subsubsection{Day 7 rates of clearance failure}

Of 56 children randomly assigned to artesunate-mefloquine on day seven of treatment, $0 \%$ had not cleared Plasmodium falciparum from their blood after 7 days of treatment with artesunate-mefloquine while of the 74 randomly allocated to generic artemether-lumefantrine $1 \%$ had not cleared Plasmodium falciparum from blood after 7 days of treatment with generic artemether-lumefantrine. A difference of $1 \%$ in rates of parasites clearance failure was observed between artesunate-mefloquine arm and generic artemether-lumefantrine arm.

Children randomly assigned to artesunate-mefloquine had zero rates of clearance failure after 7 days of treatment with artesunate-mefloquine while children randomly allocated to generic artemether-lumefantrine had $1 \%$ rates of clearance failure after 7 days of treatment with generic artemether-lumefantrine. However, the difference of $1 \%$ was not statistically significant when compared using Fisher's exact test. The Fisher's exact statistical test was used because counts in some cells were less than 5 . On day 7, there was no statistically significant difference in the rates of parasites clearance failure between artesunate-mefloquine arm and generic artemether-lumefantrine arm. Therefore, the difference in rates of parasites clearance failure was due to chance.

\subsubsection{Day 14 rates of clearance failure}

Of the 56 children randomly assigned to artesunate-mefloquine on day 14 of treatment, $0 \%$ had failed to clear Plasmodium falciparum. Similarly, of the 74 children randomly allocated to generic artemether-lumefantrine, $0 \%$ had failed to Plasmodium falciparum. There was no difference in rates of parasites clearance failure between artesunate-mefloquine arm and generic artemether-lumefantrine arm on day 14 . 


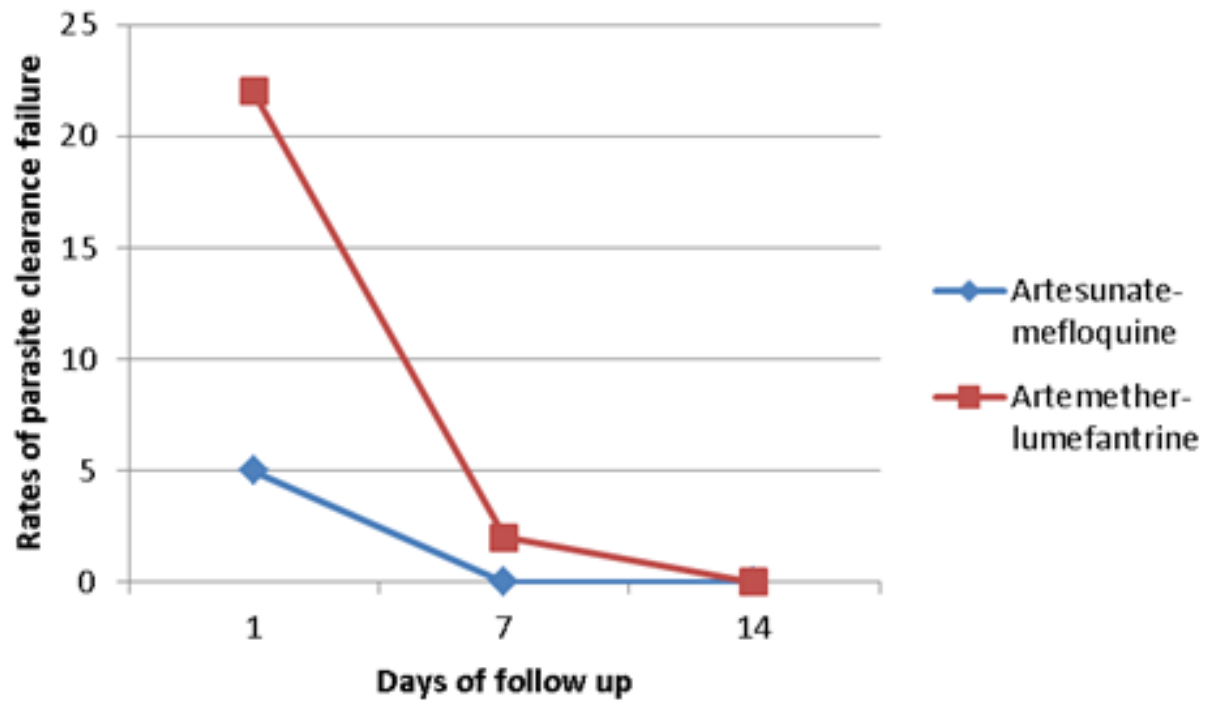

Figure 1. Comparison of rates of clearance failure

Table 2. Rates of clearance failure

\begin{tabular}{|c|c|c|c|c|c|c|}
\hline & & $\begin{array}{c}\text { Artesunate-mefloquine } \\
\%(\mathrm{n})\end{array}$ & $\begin{array}{c}\text { Artemether-lumefantrine } \\
\%(\mathrm{n})\end{array}$ & $\begin{array}{c}\text { Crude } \\
\text { OR }\end{array}$ & P-value & 95\% CI \\
\hline \multirow{4}{*}{ Day } & 1 & $5(3)$ & $22(15)$ & 0.264 & 0.020 & $0.080-0.869$ \\
\cline { 2 - 7 } & 7 & $0(0)$ & $2(1)$ & - & 0.999 & - \\
\cline { 2 - 8 } & 14 & $0(0)$ & $0(0)$ & & & \\
\hline
\end{tabular}

\section{Discussions}

This study aimed at detecting changes in sensitivity of Plasmodium falciparum as it responds to either artesunate-mefloquine or generic artemether-lumefantrine. Since this trial was not designed to use polymerase chain reaction (PCR) to distinguish between recrudescence and reinfection, accurate assessment of sensitivity of Plasmodium falciparum required that the child should not be exposed again to malaria infection during the follow up period of study, so the necessary duration of follow up was 14 days. According to Stepniewska and colleaques (6), day 14 assessment can detect high levels of resistance to antimalarial drugs. Therefore, it was thought that day 14 assessment could detect changes in the sensitivity if Plasmodium falciparum responded poorly to either generic artemether-lumefantrine or artesunate-mefloquine.

The 6 to 11 year old children was thought to be the best target population for this study because children in endemic areas age in the context of repeated infection, they gradually acquire the ability both to control parasitaemia and to resist being clinically ill (5). The ability, of 6-11 years old children, to control parasitaemia and resist being clinically ill, was assumed to make them benefit from this trial with minimal risks to their health unlike under five children and pregnant mothers. Such children with partial immunity against Plasmodium falciparum were believed to be most easily found in primary schools compared to households and health facilities.
The sample size was calculated based on day 28 polymerase chain reaction (PCR) corrected cure rates of artesunate-mefloquine that was assumed to be $96.04 \%$ (7) and artemether-lumefantrine $97.8 \%$ (4).This was the variance of the parasite clearance failure variable. It considered by how much the difference in parasite clearance rates, between artesunate-mefloquine and generic artemether-lumefaantrine, be influenced by random factors. In order to demonstrate as precisely as possible the true difference in parasite clearance failure rates, we specified type II error risk at $80 \%$ power so as to reduce the consequence of rejecting an effective medicine and type I error level of $5 \%$ to reduce the consequence of introducing an infective medicine. This study assumed a small effect size of $9 \%$ difference in rates of parasite clearance failure between the artesunate-mefloquine arm and generic artemether-lumefantrine treatment arm. This considered by how much artesunate-mefloquine should be better than generic artemether-lumefantrine. This extra effect of artesunate-mefloquine over generic artemether-lumefantrine is, according to Christensen (8) the clinical significance or least relevant difference. W.H.O as cited by Stohrer and colleaques (9) recommended a minimum sample size of 50 patients in clinical studies, if the anticipated population proportion of clinical failures is $5 \%$ (with confidence level of $95 \%$ and precision of $10 \%$ ).

Since this trial aimed at detecting changes in the context of therapeutic comparisons between artesunate-mefloquine and generic artemether-lumefantrine rather than characterization 
of parasite clearance failure profiles, parasites counts were taken once daily (after 24 hours) and on day 7 and day 14 of treatment just to make sure. Such frequency of sampling was thought to be enough for therapeutic comparisons (10).

Since the thick blood film's fixed volume method of parasite counting is less precise (10), we used an observed counting in calculating parasite densities. According to White (10), the transition from thin blood film to thick blood film does not result in a systematic error.

To reduce both systematic and random errors associated with the person performing the parasite counting, we used a three reader system: where first reader was responsible for reading the blood slide; the second reader was responsible for confirming the parasite count of the first reader; the third reader was responsible for tie breaking in case of substantial disagreement in parasite counts between the first reader and second reader. A discordance of greater than $5 \%$ in parasite counts between the first reader and second reader was considered substantial.

On day 1 , an overall $14 \%$ of the total population of both artesunate-mefloquine and generic artemether-lumefantrine had not cleared Plasmodium falciparum from their blood. This finding showed the good response of Plasmodium falciparum to both artesunate-mefloquine and generic artemether-lumefantrine after 24 hours of treatment.

However, only $5 \%$ of children randomly allocated to artesunate-mefloquine failed to clear on day 1 compared to $22 \%$ of children randomly assigned to generic artemether-lumefantrine. This finding was inconsistent with the results of a trial that was conducted by Sagara et al (7) in Mali in which the proportion of parasitaemic participants were similar between artesunate-mefloquine arm and artemether-lumefantrine arm. According to Sagara et al (7) $34.8 \%$ (81 of 233) at artesunate-mefloquine arm and $32.1 \%$ (75 of 234) at artemether-lumefantrine arm had not cleared on day 1 of treatment. So a difference of $3 \%$ was not statistically significant.

The difference between our finding and Sagara et al (7) could be due to methodological differences (equivalence trial versus superiority trial) in design and levels of endemicity (hyper endemic versus holo-endemic). In this case, our study contributed response of Plasmodium falciparum to artesunate-mefloquine versus generic artemether-lumefantrine among children in holo-endemic area. Target population could also contribute to difference in rates of parasite clearance failure. While majority of participants were of age $>5$ years in Sagara et al (7)- with possibility of including adults with fully developed immunity, our study had a clear target population of children (6-11 years) with partial immunity.

Based on day 14 assessment of treatment failure rate, W.H.O as cited by Stepniewska and colleaques (6) recommends for Africa, four policy states: Grace (0 to $4 \%)$, Alert (5 to $14 \%$ ), Action (15 to $24 \%$ ), and Change (greater or equal to $25 \%$ ). This meant that the parasite clearance failure rates of both artesunate-mefloquine and generic artemether-lumefantrine were in the grace state policy of W.H.O.

Therefore, this study concluded that Plasmodium falciparum was less likely to fail to clear as it responded to artesunate-mefloquine than to generic artemether-lumefantrine on day one of treatment. However, it was unlikely to fail to clear as it responded to both artesunate-mefloquine and generic artemether-lumefantrine on day seven and day fourteen of treatment.

It was recommended that Kisumu County's Chief Officer of Health should consider conducting regular trials to monitor and evaluate sensitivity of Plasmodium falciparum to generic artemether-lumefantrine. The Chief Officer of Health should consider carrying out more rigorous trials in order to confirm the potential of artesunate-mefloquine as another alternative treatment for uncomplicated falciparum malaria among children in Kisumu. It is recommended to the future researchers that matching be done in the future trials so as to reduce confounding age effect on clearance rates. Moreover, the future clinical trialists should consider to perform drugs label claim analysis and perhaps longer duration of follow up. Furthermore, the future researchers should study the effect of age on parasites clearance rates among older children. Finally, future researchers should consider the possibility of assessing patients' treatment seeking behaviour like self-medication.

\section{Acknowledgements}

We are very grateful to General Bior Ajang Duot, General Thomas Cirilo Suwaka, General (Dr.) Dau Aleer Abit, General (Dr) Ajak Bullen Alier, Honourable General Kuong, General (Dr.) Monywiir Arop Kuol and Colonel Jacob Malith Jok of Sudan People's Liberation Army (SPLA), Regimental Sergeant Major Miyom and the entire SPLA for their financial support.

We are also very grateful to Prof. Charles Obonyo and Dr. Micheal John Ongech of Kenya Medical Research Institute (KEMRI) for technical support, Dr. Lucy Ojwang and Dr. Onyango of Kisumu County of Kenya Ministry of Health for logistics and administrative support, Mr. Sylvester of Kisumu County of Kenya Ministry of Education, head teachers, teachers parents and children of St. Mark Nyabera, Wandiege, Buoye and Migingo government primary schools of Kisumu East Sub County for permission, moral and social support.

\section{REFERENCES}

[1] Achieng A. O., Muiruri P.,. Ingasia L. A., Opot B. H., Juma D. W., Yeda R., Ngalah B. S., Ogutu B. R., Andagalu B., Akala H. M., Kamau E., (2015) Temporal trends in prevalence of Plasmodium falciparum molecular markers selected for by artemetherelumefantrine treatment in 
pre-ACT and post-ACT parasites in western Kenya, International Journal for Parasitology: Drugs and Drug Resistance 5 : 92-99

[2] Mwai L., Kiara S. M., Abdirahman A., Pole L., Rippert A., Diriye A., Bull P., Marsh K., Borrmann S., Nzila A. (2009). In vitro activities of piperaquine, lumefantrine and dihydra-artemisinin in Kenyan Plasmodium falciparum isolates and polymorphisms in pfert and pfmdr1. Antimicrobial Agent Chemotherapy, 53 (12): 5069-5073.

[3] Zwang J., Dorsey G., Mårtensson A., d'Alessandro U., Ndiaye J., Karema C., Djimde A., Brasseur P., Sirima S. B. and Olliaro P. (2014). Plasmodium falciparum clearance in clinical studies of artesunate-amodiaquine and comparator treatments in sub-Saharan Africa, 1999-2009. Malaria Journal, 13: 114.

http://www.malariajournal.com/content/13/1/114.

[4] Ogutu, B. R., Onyango, K. O., Koskei, N., Omondi, E. K., Ongecha, J. M., Otieno, G. A., ... Juma, E. (2014). Efficacy and safety of artemether-lumefantrine and dihydroartemisinin-piperaquine in the treatment of uncomplicated Plasmodium falciparum malaria in Kenyan children aged less than five years: results of an open-label, randomized, single-centre study. Malaria Journal, 13, 33. http://doi.org/10.1186/1475-2875-13-33

[5] Zhou G., Afrane Y. A., Vardo-Zalik A. M., Atieli H., Zhong D., Wamae P., Himeidan Y. E., Minakawa N., Githeko A. K., Yan G. (2011). Changing patterns of malaria epidemiology between 2002 and 2010 in western Kenya: the rise and fall of malaria. PLos ONE 6(5):e20318

[6] Stepniewska K., Taylor W. R. J., Mayxay M., Price R., Smithuis F., Guthmann J., Barnes K., Myint H. Y., Adjuik M., Olliaro P., Pukrittayakamee S., Looareesuwan S., Hien T. T., Farrar J., Nosten F., Day N. P. J., White N. J. (2004). In vivo assessment of drug efficacy against Plasmodium falciparum malaria: duration of follow -up. Antimicrobial, Agent and Chemotherapy, 48 (11): 4271-4280.

[7] Sagara, I., Diallo, A., Kone, M., Coulibaly, M., Diawara, S. I., Guindo, O., ... Doumbo, O. K. (2008). A Randomized Trial of Artesunate-Mefloquine versus Artemether-Lumefantrine for Treatment of Uncomplicated Plasmodium falciparum Malaria in Mali. The American Journal of Tropical Medicine and Hygiene, 79(5), 655-661.

[8] Christensen E. (2007). Methodology of superiority vs. equivalence trials and non-inferiority trials. Journal of Hepatology 46: 947-954.

[9] Stohrer J. M., Dittrich S., Thongpaseuth V., Vanisaveth V., Phetsouvanh R., Phompida S., F., Christophel E., Lindegardh N., Annerberg A., Jelinik T. (2004). Therapeutic efficacy of artemether-lumefantrine and artesunate-mefloquine for the treatment of uncomplicated malaria Plasmodium falciparum malaria in Luang Namtha Province, Lao People's Democratic Republic. Tropical Medicine and Internation Health 9 (11): 1175-1183.

[10] White N. J. (2011). The Parasite Clearance Curve. Malaria Journal, 10: 278. 